\title{
Gamma-ray bursts vs. afterglows
}

\section{J.I. Katz}

Department of Physics and McDonnell Center for the Space Sciences, Washington University, St. Louis, MO. 63130, U.S.A. e-mail: katz@wuphys.wustl.edu

Received December 18, 1998; accepted March 12, 1999

\begin{abstract}
When does a GRB stop and its afterglow begin? A GRB may be defined as emission by internal shocks and its afterglow as emission by an external shock, but it is necessary to distinguish them observationally. With these definitions irregularly varying emission (at any frequency) must be the GRB, but smoothly varying intensity is usually afterglow. The GRB itself and its afterglow may overlap in time and in frequency, and distinguishing them will, in general, require detailed modeling.
\end{abstract}

Key words: gamma-ray bursts — shock waves

At first glance there appears to be little difficulty in distinguishing between GRB and their afterglows. GRB were discovered in 1972, and are observed in gamma-rays with detectors most sensitive to photons in the range $100-1000 \mathrm{keV}$. The observed durations of GRB range from milliseconds to $\sim 1000 \mathrm{~s}$. Afterglows were first observed in 1997 in the radio, visible and X-ray bands, and have durations of hours to months. These appear to be very different phenomena, although causally associatedafterglows follow GRB.

This phenomenological distinction between GRB and their afterglows is likely to become insufficient. It is based on the observations of GRB and of afterglows by two distinct classes of instruments with distinct (and largely nonoverlapping) sensitivities:

1. GRB detectors have broad angular acceptance and little sensitivity below $30 \mathrm{keV}$. Their angular acceptance implies high background levels. This makes them comparatively insensitive to steady sources of low flux, although very sensitive to transients of low fluence. These properties are not failures of instrument design; rather, they represent the optimal adaptation of detector technology to the observation of unpredictable brief transients of high peak flux but low fluence (compared to a steady source integrated over a long time).

Send offprint requests to: J.I. Katz
2. Afterglows are detected with instruments which are sensitive to steady sources of low flux and known position. Observing such sources is the usual problem in astronomy, and these are conventional astronomical instruments, whose sensitivity depends on long temporal integration and high angular resolution. Their resolution discriminates against background. They cannot find unpredictable transients because their high resolution limits their angular acceptance, but once steered to an afterglow by a GRB detector they are sensitive to sources of low flux but long duration (and therefore of comparatively large fluence).

This instrumental distinction between GRB and their afterglows will not survive when the gap between the two classes of instruments is bridged. To some extent, this has already happened, as the X-ray detectors on BeppoSAX are able to detect $\mathrm{X}$-ray emission during the brief gammaray duration of bright GRB within their field of view. The X-ray and soft gamma-ray bands overlap around $10-30 \mathrm{keV}$ (the distinction between them is largely a matter of definition), and X-ray emission during the detected gamma-ray emission is generally considered to be just the low frequency extrapolation of the gamma-ray emission (although there is a lively controversy concerning the validity and interpretation of this extrapolation; Katz 1994a; Preece et al. 1996; Cohen et al. 1997; Preece et al. 1998).

The detection of simultaneous visible counterparts has long been the holy grail of GRB research. Instruments are rapidly improving in sensitivity and speed of response, and the prospect of rapid $(<10 \mathrm{~s})$ dissemination of accurate GRB coordinates (for example, from HETE-2) makes it likely that they will be observed soon.

The phenomenological distinction between GRB and their afterglows will disappear when the temporal gap between simultaneous and delayed (by hours to days) observations is bridged. Once the simultaneous radiation of a GRB is detected, continuing to stare at that point on the sky will produce a continuous intensity history interrupted only by dawn, bad weather or Earth occultation. 
Will there then be any possibility of distinguishing between GRB and their afterglows, or any purpose in doing so? I wish to argue that the answer to both these questions is yes, provided the terms GRB and afterglow are redefined as indicating the physical processes which produce the radiation.

There is no doubt that GRB involve relativistic outflow from a central source of energy, and that the observed radiation is produced in optically thin (except at radio frequencies) regions far from the central source. In order to tap the kinetic energy of relativistic matter it must exchange momentum with some other matter or radiation, either nearly at rest in a local observer's frame or also moving relativistically. The former case is called an external shock, and the matter at rest is generally assumed to be either the surrounding interstellar medium or a non-relativistic outflow produced by the GRB's progenitor. The latter case is called an internal shock, and the interaction is assumed to be between different portions of the relativistic outflow, produced at different times and with differing Lorentz factors. Although the term "shock" is generally used, it is neither necessary nor demonstrated that a shock occurs; streams of low density matter may interpenetrate, exchanging momentum more gradually as a consequence of plasma instability (such an instability is also required for a shock, which must be collisionless because of the low densities).

In at least some GRB most of the early gamma-ray emission is produced by internal shocks. These GRB consist of several sharp and clearly separated subpulses, often with intensity dropping to background levels between the subpulses. Fenimore et al. (1996) and Sari \& Piran (1997) showed that such temporal behavior cannot be produced by an external shock of plausible efficiency, no matter how clumpy the external medium, thus refuting the original argument (Rees \& Mészáros 1992; Katz 1994b) for external shock models, that interaction with a heterogeneous medium can explain how a single class of similar collapse or coalescence events could produce the observed "zoo" of diverse GRB pulse profiles. The radiation of these multipeaked GRB can only be explained by internal shock (Rees \& Mészáros 1994) models, in which the variability is attributed to modulation of a longer-lived source of energy (Katz 1997). Additional evidence for the internal shock origin of multi-peaked GRB was presented at this meeting by Fenimore \& Ramirez-Ruiz (1999) and by Quilligan et al. (1999) who found that the properties of their subpulses do not evolve through a burst, suggesting that subpulses are independent events, in effect individual GRB. This is inconsistent with external shock models, in which the characteristic radii and time scales monotonically increase.

Internal shocks are rather inefficient $(\sim 20 \%$ for typical parameters) in dissipating (a precondition for radiating) the kinetic energy of relativistic outflows. Because no GRB occurs in perfect vacuum, even if it were to occur in an intergalactic medium, there is always matter for external interaction. The efficiency of radiation by this external interaction may be low, particularly if the ambient density is low. It is natural to associate this external interaction (or shock) with the phenomenologically defined afterglow because its duration is predicted to be much longer than that of internal shocks, and because the smooth single-peaked behavior of afterglows observed (to date) is consistent with that predicted (Katz 1994a, 1994b) for external shocks.

I suggest that it is useful to define GRB as the radiation produced by internal shocks and afterglows as the radiation produced by external shocks. Then, instead of arguing about nomenclature, we can engage in a more scientifically productive discussion about the physical origin of the various components of the observed radiation. How can they be distinguished?

A spiky temporal profile is an unambiguous indicator of an internal shock, but that rule does not answer all questions. There are GRB with smooth single-peaked profiles, which can be explained by either internal or external shocks, The observed duration of some GRB is $\sim 1$ hour, and others may last a day or longer (Katz 1997), so that duration is also not sufficient to distinguish GRB from their afterglows. In some bursts, the internal and external shock emission may overlap in both spectrum and in time.

Unfortunately, there appears to be no general rule for distinguishing external from internal shock emission. The predicted asymptotic spectra with and without radiative cooling (Cohen et al. 1997) do not distinguish between internal and external shocks. Making this distinction will require detailed spectral and temporal modeling to associate different spectral components which are produced by the same physical process. Identifying the physical origin of each will require hydrodynamic modeling of the source. This is likely to be a formidable task.

Acknowledgements. This work was supported in part by NSF AST 94-16904.

\section{References}

Cohen E., et al., 1997, ApJ 488, 330

Fenimore E.E., Madras C.D., Nayakchin S., 1996, ApJ 473, 998

Fenimore E.E., Ramirez-Ruiz E., 1999 (these proceedings)

Katz J.I., 1994a, ApJL 432, L107

Katz J.I., 1994b, ApJ 422, 248

Katz J.I., 1997, ApJ 490, 633

Preece R.D., et al., 1996, ApJ 473, 310

Preece R.D., et al., 1998, ApJL 506, L23

Quilligan F., Hurley K.J., McBreen B., Hanlon L., 1999 (these proceedings)

Rees M.J., Mészáros P., 1992, MN 258, 41P

Rees M.J., Mészáros P., 1994, ApJL 430, L93

Sari R., Piran T., 1997, ApJ 485, 270 